\title{
Detecting Ultra High Energy Neutrinos by Upward Tau Airshowers and Gamma Flashes
}

\author{
Daniele Fargion ${ }^{1}$ \\ Physics Department and INFN, Rome University 1, \\ Pl.A.Moro 2, 00185, Rome, Italy
}

\begin{abstract}
Air-showers are the best trace of rarest Ultra High Energy neutrinos UHE $\nu_{\tau}, \bar{\nu}_{\tau}$ and $\bar{\nu}_{e}$ at PeV and higher energy. $\tau$ Air-showers may generate billion times amplified signals by their secondaries. Horizontal amplified $\tau$ air-showers by $\nu_{\tau} N$ and UHE $\bar{\nu}_{e} e$ at PeV emerging from mountain chain might be the most powerfull imprint. Upward UHE $\nu_{\tau} N$ interaction on Earth crust at horizontal edge and from below, their consequent UHE $\tau$ air-showers beaming toward high mountains should flash $\gamma, \mu, \mathrm{X}$ and optical detectors on the top. Upward $\tau$ air-shower may hit nearby satellite flashing them by short, hard, diluted $\gamma$-burst at the edge of Gamma Ray Observatory BATSE threshold. We identify these events with recent (1994) discovered upward Terrestrial Gamma Flashes (TGF) and we probed their UHE $\tau$ - UHE $\nu_{\tau}$ origin. From these TGF data approximated UHE $\nu_{\tau}$ flux and $\Delta m_{\nu_{\mu} \nu_{\tau}}$ sever lower bound are derived. Partial TGF Galactic signature is also manifest within known 47 TGF events at $\simeq 2 \cdot 10^{-3}$ probability. Well known $X-\gamma-\mathrm{TeV}$ active galactic and extragalactic sources have found probable counterpart in TGF arrival directions. Detection of elusive UHE $\nu_{\tau}$ seem finally achieved.
\end{abstract}

\section{The UHE $\bar{\nu}_{e}, \nu_{\tau}, \bar{\nu}_{\tau}$ interactions and their UHE $\tau$ secondary}

Ultra high energy astrophysical neutrino $(\mathrm{UHE} \nu)$ from $\mathrm{PeVs}\left(\gtrsim 10^{15} \mathrm{eV}\right)$ up to $\left(10^{18} \mathrm{eV}\right) \mathrm{EeV}$ and GZK cut off energies $\left(\gtrsim 10^{19} \mathrm{eV}\right)$ might be traced by $\tau$ induced air showers and by their millions to hundred billions multiplicity in secondaries particles, $\left(N_{\mu} \sim 10^{6}\left(\frac{E_{\nu}}{P e V}\right), N_{X} \sim 10^{10}\left(\frac{E_{\nu}}{P e V}\right), N_{o p t} \gtrsim 10^{11}\left(\frac{E_{\nu}}{P e V}\right)\right)$. Indeed astrophysical PeVs UHE anti-neutrino electrons, $\bar{\nu}_{e}$, near the Glashow W resonance peak, $E_{\overline{\nu_{e}}}=M_{W}^{2} / 2 m_{e} \simeq 6.3 \cdot 10^{15} \mathrm{eV}$, (dominant over expected UHE $\mathrm{PeV}$ atmospheric neutrino signals), may be observable by their secondary horizontal $\tau$ air showers originated by UHE chain reaction $\bar{\nu}_{e}+e \rightarrow W^{-} \rightarrow \bar{\nu}_{\tau}+\tau^{-}$ inside the concrete rock of a high mountain. Also UHE $\nu_{\tau}, \bar{\nu}_{\tau}$ at $\left(10^{16}-10^{17} \mathrm{eV}\right)$ interacting with nuclear matter $\left(\nu_{\tau} N\right)$ must be observable if flavor mixing $\nu_{\mu} \leftrightarrow \nu_{\tau}$ take place as shown by Superkamiokande data, because of huge astrophysical distances respect to oscillation ones.

Therefore UHE $\nu_{\tau}$ and $\overline{\nu_{\tau}}$ may be converted and they may reach us from high energy galactic sources, as pulsars, SNRs, black holes or galactic SGRs microquasars, as well as from powerful extragalactic AGNs, quasars or GRBs, even for any small mass mixing, $\left(\Delta m_{i j}^{2} \sim 10^{-4} \mathrm{eV}^{2}\right)$ or any high (GZK) energy because of the large galactic (Kpcs) and extreme cosmic (Mpcs) distances: 


$$
L_{\nu_{\mu}-\nu_{\tau}}=4 \cdot 10^{-3} p c\left(\frac{E_{\nu}}{10^{16} \mathrm{eV}}\right) \cdot\left(\frac{\Delta m_{i j}^{2}}{\left(10^{-2} \mathrm{eV}\right)^{2}}\right)^{-1}
$$

Rare upward UHE $\tau$, born by $\nu_{\tau}$ and $\overline{\nu_{\tau}}$ nuclear (or rare $\overline{\nu_{e}}-e$ interactions near the upward earth surface), may escape outside on air where they may spontaneously decay triggering upward vertical, oblique or near horizontal $\tau$ air showers. The vertical ones (by small nadir angle) occur preferentially at low energies nearly transparent to the Earth $\left(E_{\nu} \sim 10^{15}-10^{16} \mathrm{eV}\right)$. The oblique $\tau$ air showers whose arrival directions have large nadir angle, are related mainly to higher energy $\nu_{\tau}$, or $\overline{\nu_{\tau}}$ nuclear interactions $\left(E_{\overline{\nu_{\tau}}} \geq 10^{17}-10^{20} \mathrm{eV}\right)$. Indeed these horizontal - upward UHE $\nu_{\tau}$ cross a smaller fraction of the Earth volume and consequently they suffer less absorption toward the horizon.

These huge horizontal or upward air-shower signals being at least million to billion times more abundant than the original and unique UHE $\tau$ or UHE $\mu$ track in underground $\mathrm{Km}$ cube detectors are much easier to be discovered with no ambiguity. We remind that long tracks in $\mathrm{km}^{3}$ detectors are mostly noisy signals by $\mathrm{TeVs}$ to tens of $\mathrm{TeVs}$ muons secondaries generated by atmospheric neutrinos born by common cosmic ray interactions in upper atmosphere. The $\tau$ air shower is analogous to the Learned and Pakwasa (1995) "double bang" in underground neutrino detectors. The novelty of the present "one bang in" (the rock) - "one bang out" (the air) lays in the self-triggered explosive nature of $\tau$ decay in flight and its consequent huge amplified air shower signal at a characteristic few Kms distance. Detectable horizontal gamma bursts (mainly bremsstrahlung photons) are among the most abundant signal. The source of UHE $\nu_{\tau}$, since Super Kamiokande evidence of neutrino flavour mixing, must be as abundant as muon $\nu_{\mu}$ ones.

Moreover the expected $\nu_{\tau}$ signals, by their secondary tau tracks at highest cosmic ray energy window $1.7 \cdot 10^{21} \mathrm{eV}>E_{\tau}>1.6 \cdot 10^{17} \mathrm{eV}$, must exceed the corresponding $\nu_{\mu}$ (or muonic) ones, making UHE $\nu_{\tau}$ above $0.1 \mathrm{EeV}$ the most probable UHE signal. Indeed, the Lorentz-boosted tau range length grows (linearly) above muon range, for $E_{\tau} \geq 1.6 \cdot 10^{8} \mathrm{GeV}$; (see Fig (1) eq.3): the tau track reaches its maxima extension, bounded not by pair production (eq. 2), but by growing nuclear electro-weak interactions (eq. 4), $R_{\tau_{\max }} \simeq 191 \mathrm{Km}$, at energy $E_{\tau} \simeq 3.8 \cdot 10^{9} \mathrm{GeV}$.

$$
\begin{gathered}
R_{R_{\tau}} \cong 1033 K m\left(\frac{\rho_{r}}{5}\right)^{-1} \cdot\left\{1+\frac{\ln \left[\left(\frac{E_{\tau}}{10^{8} \mathrm{GeV}}\right)\left(\frac{E_{\tau}^{\mathrm{min}}}{10^{4} \mathrm{GeV}}\right)^{-1}\right]}{\left(\ln 10^{4}\right)}\right\} . \\
R_{\tau_{o}}=c \tau_{\tau} \gamma_{\tau}=5 \mathrm{Km}\left(\frac{E_{\tau}}{10^{8} \mathrm{GeV}}\right) . \\
R_{W_{\tau}}=\frac{1}{\sigma N_{A} \rho_{r}} \simeq \frac{2.6 \cdot 10^{3} \mathrm{Km}}{\rho_{r}}\left(\frac{E_{\tau}}{10^{8} \mathrm{GeV}}\right)^{-0.363} .
\end{gathered}
$$


At this peak the tau range is nearly 20 times longer than the corresponding muon range (at the same energy) implying, for comparable fluxes, a ratio 20 times larger in $\nu_{\tau}$ over $\nu_{\mu}$ detection probability. This dominance, may lead to a few rare spectacular event a year (if flavor mixing occurs) preferentially in horizontal plane in underground $\mathrm{Km}^{3}$ detectors. The Earth opacity at those UHE regimes at large nadir angles (nearly horizontal, few degree upward direction) is exponentially different for UHE muons respect to tau at GZK energies (corresponding to $500 \mathrm{Kms}$ UHE Tau lenghts), making the muon/tau flux ratio of such lenghts severely (half billion time) suppressed.

UHE $\nu$ above GZK are transparent to BBR cosmic photons and they may easily reach us from far cosmological distances. Therefore the puzzle of UHECR above GZK cut off may be solved assuming that neutrinos (possibly of heaviest Muon/Tau nature)share a light mass of few $\mathrm{eV}$, in the frame-work of Hot Dark Matter halos clustered around galaxies. Such light neutrinos may form a huge hidden dark calorimeter able to beam dump UHE $\nu$ via $Z$ (s-channel), via virtual $W$ ( $t$ channel $)$ or $\mathrm{W}$ pair productions. The corresponding cross sections for such $\nu \nu$ interactions are shown in Fig. 2; their secondaries may be final UHE anti-protons (or anti-neutrons) or UHE protons (or neutrons) (Fargion,Mele,Salis 1997-1999) responsible of final observed UHECR above GZK cut off. The interaction efficency by relic light neutrinos via UHE $\nu$ at GZK cut off is thousands times larger than UHE $\nu$ interactions on Earth atmosphere and/or direct UHECR (nucleons,nuclei) propagations above GZK distances. Therefore light neutrino mass may explain both hot dark matter and UHECR above GZK (as well as their recent clustering in triplets or doublets). Just to emphasize the $\nu$ mass roles in high energy astrophysics, we remind the important case of a SN $\mathrm{MeVs}$ neutrino burst arriving slowed by its mass relativistic flight and its delayed arrival from far SN (galactic or better extragalactic) events respect to the massless (prompt coeval) gravitational waves. The expected time delay between the massless graviton wave burst (by supernova quadrupole emission at distance $\mathrm{L}$ and the $\nu_{e}$ neutronization neutrino burst), will be an additional tests test to the elusive mass detection: $\Delta t \sim 50 \sec \left(\frac{E_{\nu}}{5 M e V}\right)^{-2}\left(\frac{m_{\nu}}{5 e V}\right)^{2}\left(\frac{L}{M p c}\right)$. (Fargion 1981). Let us remind that massive neutrino imply new right handed interactions in early Universe (Antonelli, R.Konoplich, D.Fargion 1981) and multifluid gravitational clustering during galaxy formation epochs (D.Fargion 1981, 1983).

UHE Tau $E_{\tau} \geq 10^{5} \mathrm{GeV}-5 \cdot 10^{7} \mathrm{GeV}$ air-shower in front of high mountains chains will be easily induce peculiar horizontal UHE $\tau$ decay beyond a thick mountain (Fargion, Aiello, Conversano 1999). The high mountain act as a clever filter:

a) as a wide angle screen of undesirable horizontal Ultra High Energy Cosmic Rays (UHECR) (electro-magnetic shower, secondary Cherenkov photons and muons),b) as a calorimeter for UHE $\nu_{\tau}, \bar{\nu}_{\tau}$ and $\bar{\nu}_{e}, \mathrm{c}$ ) as a distance meter correlating tau relativistic track and birth and its air-shower opening distance from the mountain with UHE tau original energy. d) as an unique source, by tau electromagnetic showering, of horizontal rich, sharp, $\mu$ second burst $\gamma$, X flash, electron pairs and Cherenkov showers source. e) as an unique source, by tau hadronic 
showering, of additional horizontal dense muon pairs sharp bundle burst.

An hybrid detector (gamma/optical)would get precise signal and arrival direction. Because of the different neutrino interactions with energy and flavors it will be possible to estimate, by stereoscopic, directional and time structure signature, the spatial air-shower origination in air, the primary tau distance decay from the mountain (tens of meter for PeVs UHE $\bar{\nu}_{e}$ and nearly hundred meters up to Kms for UHE $\left.\nu_{\tau}, \bar{\nu}_{\tau}\right) E_{\tau} \geq 10^{5} \mathrm{GeV}, 5 \cdot 10^{7} \mathrm{GeV}$, the consequent most probable original UHE tau range and energy. Additional energy calibration may be derived sampling shower intensities.

Hundreds of detectors in deep wide valley would be necessary to get tens taus of event a year.

Screening by undesirable lateral or downward noisy cosmic rays or natural radiation is possible by directional and time clustering filter; therefore the induced $\bar{\nu}_{e} e \rightarrow \tau$ air shower even in absence of $\nu_{\mu} \leftrightarrow \nu_{\tau}$ oscillation should be identified and detectable soon. Its unique $\bar{\nu}_{e}$ origin is marked by the peaked $\mathrm{W}$ resonance, and by the small mountain $\bar{\nu}_{e}$ opacity and its high neutrino cross-section. Its identity is marked by the expected fine tuned $\mathrm{PeV}$ energy at $\mathrm{W}$ peak and the tau air-shower birth place near (a hundred meter) the mountain wall.

More copious ( $>5$ times more) events by $\mathrm{PeV}$ up to tens $\mathrm{PeV} \quad \nu_{\tau} N$ interaction occur if, as most of us believe, $\nu_{\mu}$ oscillate in $\nu_{\tau}$.

It will be also possible to discover UHE $\tau$, by observing the upward tau airshower arriving from hundred Kilometers away (near horizontal edges) from high mountains, high balloon and satellites; such UHE tau created within a wide (tens thousands to millions square $\mathrm{km}^{2}$ wide and hundred meter UHE Tau depth in Earth crust) target would discover easely UHE $\nu_{\tau}, \bar{\nu}_{\tau}$ neutrinos at $\mathrm{PeV}$ up to EeV energies and above, just within the mysterious GZK frontiers. The discover will need capable gamma, optical and mainly muon bundle detectors within present technology as studied elsewhere.

From the same highest mountains, balloons and near orbit satellite, looking more downward toward the Earth it is possible to discover more frequent but lower energetic astrophysical $\simeq \mathrm{PeV}$ - tens $\mathrm{PeV}$ neutrinos still nearly transparent to the Earth volumes and (Gandhi et al. 1998),(see Fig.3).

The UHE neutrinos $\overline{\nu_{e}}, \nu_{\mu} \bar{\nu}_{\mu}$ are default and expected UHECR $\left(\gtrsim 10^{16} \mathrm{eV}\right) \mathrm{sec}-$ ondary products near AGN or microquasars by common photo-pion decay relics by optical photons nearby the source (PSRs, AGNs) $\left(p+\gamma \rightarrow n+\pi^{+}, \pi^{+} \rightarrow\right.$ $\mu^{+} \nu_{\mu}, \mu^{+} \rightarrow e^{+} \nu_{e} \bar{\nu}_{\mu}$ ), or by proton proton scattering in galactic interstellar matter. The maximal observational distances from mountains, baloons or satellites, may reach $\sim 110 \mathrm{Km}(h / K m)^{\frac{1}{2}}$ toward the horizon, corresponding to a UHE $\tau$ energy $\sim 2 \cdot 10^{18} \mathrm{eV}(h / K m)^{\frac{1}{2}}$. Therefore we propose to consider such upward shower nearly horizontal detection from high mountains to test this highest $\nu_{\tau} \overline{\nu_{\tau}}$ energy window almost opaque to Glashow UHE $\overline{\nu_{e}}$ fluxes. 


\section{Upward $\tau$ air shower in Terrestrial Gamma Flash}

The expected downward muon number of events $N_{e v}\left(\bar{\nu}_{e} e \rightarrow \bar{\nu}_{\mu} \mu\right)$ in the resonant energy range, in $\mathrm{Km}^{3}$, [Table 7, The Gandhi et all,1998] was found to be $N_{e v}=6$ a year. One expect a comparable number of reactions $\left(\bar{\nu}_{e} e \rightarrow \bar{\nu}_{\tau} \tau\right)$. However the possible presence of primordial $\nu_{\tau}, \overline{\nu_{\tau}}$ by flavor mixing and $\nu_{\tau}, \overline{\nu_{\tau}} N$ charged current interactions lead to a factor 5 larger rate, $N_{e v}=29$ event/year.

If one immagines a gamma/optical detector at $5 \mathrm{~km}$ far in front of the Alps Argentier mountains (size $10 \mathrm{~km}$, height $1 \mathrm{~km}$ ) one finds a $\tau$ air shower volume observable within a narrow beamed cone (Moliere radius $\sim 80 \mathrm{~m} /$ distance $\sim 5 \mathrm{Km}):\left(\Delta \theta \sim 1^{o}, \Delta \Omega \sim 2 \cdot 10^{-5}\right)$ and an effective volume $V_{\text {eff }} \simeq 9 \cdot 10^{-5}$ $\mathrm{Km}^{3}$ for each observational detector (Fargion 2000). Each one is comparable to roughly twice a Super Kamiokande detector. We expect, following AGN - SS91 model [The Gandhi et all,1998] a total rate of: $(6)\left(\overline{\nu_{e}} e\right)+(29)\left(\nu_{\tau} N\right)=35$ UHE $\nu_{\tau}$ event/year $/ \mathrm{Km}^{3}$; at energies above $3 \mathrm{PeV}$ we may expect a total rate of $\mathrm{N}_{e v} \sim 158$ event/year in this Alps Argentiere mountains valley and nearly 3.2 $\cdot 10^{-3}$ event/year for each detector. In a first approximation, neglecting Earth opacity, it is possible to show that the Earth volume observable from the top of a mountain at height $h$, due to UHE $\tau$ at $3 \mathrm{PeV}$ crossing from below, is approximately $\mathrm{V} \approx 5 \cdot 10^{4} \mathrm{Km}^{3}\left(\frac{h}{K m}\right)\left(\frac{E_{\tau}}{3 \mathrm{PeV}}\right)$. The upward shower would hit the top of the mountain. For the same $\tau$ air shower beaming $\left(\Delta \theta \sim 1^{o}, \Delta \Omega \sim 2 \cdot 10^{-5}\right)$ we derive now an effective volume $\sim 1 \mathrm{Km}^{3}$. Therefore a detector open at $2 \pi$ angle on a top of a $2 \mathrm{Km}$ height mountain may observe nearly an event every two month from below the Earth. The gamma signal above few $\mathrm{MeV}$ would be (depending on arrival nadir angle) between $3 \cdot 10^{-2} \mathrm{~cm}^{-2}$ (for small nadir angle) to $10^{-5} \mathrm{~cm}^{-2}$ at far distance within $3 \mathrm{PeV}$ energies. A contemporaneous (microsecond) optical flash ( $\gtrsim 300 \div 0.1 \mathrm{~cm}^{-2}$ ) must occur. Keeping care of the Earth opacity, at large nadir angle $\left(\gtrsim 60^{\circ}\right)$ where an average Earth density may be assumed $(<\rho>\sim 5)$ the transmission probability and creation of upward UHE $\tau$ is approximately

$$
P\left(\theta, E_{\nu}\right)=e^{\frac{-2 R_{E a r t h} \cos \theta}{R_{\nu_{\tau}}\left(E_{\nu}\right)}}\left(1-e^{-\frac{R_{\tau}\left(E_{\tau}\right)}{R_{\nu \tau}\left(E_{\nu}\right)}}\right) .
$$

This value, at $\mathrm{PeV}$ is a fraction of a million.

The corresponding angular integral effective volume observable from a high mountain (or baloon) at height $h$ (assuming a final target terrestrial density $\rho=3)$ is:

$$
V_{e f f} \approx 0.3 K m^{3}\left(\frac{\rho}{3}\right)\left(\frac{h}{K m}\right) e^{-\left(\frac{E}{3 P e V}\right)}\left(\frac{E}{3 P e V}\right)^{1.363}
$$

This rate is quite large and the expected $\tau$ air airshower signal (gamma burst at energies $\gtrsim 10 \mathrm{MeV}$ ) should be $\phi_{\gamma} \simeq 10^{-4} \div 10^{-5} \mathrm{~cm}^{-2}$, while the gamma flux at $\left(\sim 10^{5} \mathrm{eV}\right)$ or lower energies (from electron pair bremsstrahlung) may be two order of magnitude larger.

The optical Cherenkov flux is large $\Phi_{\text {opt }} \approx 1 \mathrm{~cm}^{-2}$. The tau upward air showers 
born in a narrow energy window, $10^{15} \mathrm{eV} \lesssim E_{\nu} \lesssim 5 \cdot 10^{16} \mathrm{eV}$ (Fig.3) may penetrate high altitude leaving rare beamed upward gamma shower bursts whose sharp ( hundreds $\mu$ sec because of the hundred kms high altitude shower distances) time structure and whose hard $\left(\gtrsim 10^{5} \mathrm{eV}\right)$ spectra may hit near terrestrial satellites. We claim (Fargion 2000) that such gamma upward events originated by tau air showers produce gamma bursts at the edge of GRO-BATSE sensitivity threshold. In particular we argue that very probably such upward gamma events have been already detected since April 1991 as serendipitious sharp $\left(\lesssim 10^{-3} \mathrm{sec}\right)$ and hard $\left(z 10^{5} \mathrm{eV}\right.$ ) BATSE gamma triggers originated from the Earth and named consequently as Terrestrial Gamma Flashes (TGF). The visible Earth surface from a satellite, like BATSE, at height $h \sim 400 \mathrm{Km}$ and the consequent effective volume for UHE $\nu_{\tau} N$ PeVs interaction and $\tau$ air shower beamed within $\Delta \Omega \sim 2 \cdot 10^{-5} \mathrm{rad}^{2}$ is: (note $\langle\rho\rangle \simeq 1.6$ because $70 \%$ of the Earth is covered by seas) $V_{\text {eff }}=V_{T O T} \Delta \Omega \simeq 60 \mathrm{Km}^{3}$. The effective volume and the event rate should be reduced, at large nadir angle $\left(\theta>60^{\circ}\right)$ by the atmosphere depth and opacity (for a given $E_{\tau}$ energy). Therefore the observable volume may be reduced approximately to within $15 \mathrm{Km}^{3}$ values and the expected UHE PeV event rate is

$$
N_{e v} \sim 150 \cdot e^{-\left(\frac{E_{\tau}}{3 P e V}\right)}\left(\frac{E_{\tau}}{3 P e V}\right)^{1.363}\left(\frac{h}{400 K m}\right) \quad \frac{\text { events }}{\text { year }}
$$

The TGF signals would be mainly $\gamma$ at flux $10^{-2} \mathrm{~cm}^{-2}$ at $\mathrm{X}$ hundred keV energies. The observed TGF rate is lower by nearly an order of magnitude, and this suggests higher $E_{\nu}$ energies (to overcome BATSE threshold) and consequently small additional probability suppression leading to observed TGF events rate. However since 1994 (Fishman et al.) TGF understanding of presently known 75 records over nearly eight thousand BATSE triggers is based on an unexpected and mysterious high latitude lightening of geophysical nature (the so called "Sprites" or "Blue Jets"). We do not believe in that interpretation. We notice that among the 75 records only 47 are published in their details, while 28 TGF are still unpublished. Their data release is therefore urgent and critical. While Blue Jets might be in principle triggered by upward tau air showers in the atmosphere (a giant "Wilson" room) we believe they are not themselves source of TGF. In particular their observed characteristic propagation velocity $(\lesssim 100$ $\mathrm{Km} / \mathrm{s}$ ) from distances $\sim 500 \mathrm{Km}$, disagree with short TGF millisecond timing and would favor a characteristic TGF time of few seconds.

Moreover TGF data strongly disfavor by its hard spectra the terrestrial Sprites connection. On the contrary the expected UHE tau upward air showers lead to a gamma burst flux, spectra, and fine time structure fluence in agreement with the observed TGF ones and in agreement with the expected flux models. The correlations of these clustered TGFs directions toward

(1) well known and maximal powerful galactic and extra-galactic sources either at $\mathrm{TeV}, \mathrm{GeV}-\mathrm{MeV}, \mathrm{X}$ band ,(2) recent first anisotropy discovered on UHECR at EeV by AGASA, (see Fig.4, and Hayoshida et al. 1999) (3) the Milky Way Galactic Plane, support and make compelling the TGF identification as sec- 
ondary gamma burst tail of UHE $\tau$ induced upward air shower. The present TGF- $\tau$ air-shower identification could not be produced by UHE $\bar{\nu}_{e}$ charged current resonant event at $\left(E_{\overline{\nu_{e}}}=M_{W}^{2} / 2 m_{e}=6.3 \cdot 10^{15} \mathrm{eV}\right)$, and therefore it stand for the UHE $\nu_{\tau} \overline{\nu_{\tau}}$ existence. Consequently it gives support to the Superkamiokande evidences for $\nu_{\mu} \leftrightarrow \nu_{\tau}$ flavor mixing from far PSRs or AGNs sources toward the Earth. At the present the very probable $\nu_{\tau} \overline{\nu_{\tau}}$ source of TGFs and their probable partial galactic location infer a first lower bound on $\Delta_{m_{\nu_{\mu} \nu_{\tau}}}(L<4$ $\left.\mathrm{Kpc}, \Delta_{m_{\nu_{\mu} \nu_{\tau}}}>10^{-8} \mathrm{eV}^{2}\right)$ and it offers a first direct test of the same existence of the last missing, yet unobserved, fundamental neutral lepton particle: $\nu_{\tau}$ and $\overline{\nu_{\tau}}$.

\section{References}

1. J. G. Learned \& Pakvasa, S. 1995, Astropart. Phys. 3, 267 89, 833

2. Gandhi, R., Quigg, C., Reno, M. H., Sarcevic, I., Phys. Rev. D 58, 1998.

3. Fargion, D. Salis, A. Proc. 25th ICRC. HE-4-6, 153-156 (1997).

4. D. Fargion, A. Salis, B. Mele, astro-ph/9710029, Ap. J. 517 ,725-733, 1999.

5. D. Fargion, Lett.Nuovo Cimento Vol. 31, No. 199, (1981)

6. D. Fargion ,Nuovo Cimento Vol.65B, No. 316, (1981); F. Antonelli, R. Konoplich and D. Fargion, Lett. Nuovo Cimento Vol. 32, No. 289, (1981).

7. D. Fargion, Nuovo Cimento Vol. 77B, No. 111, (1983)

8. D. Fargion, A. Aiello, R. Conversano , 26th ICRC Salt Lake, USA, HE6.1.10, 396-398 (1999); astro-ph/9906450.

9. D. Fargion, astro-ph/9704205/0002453,submitted to Ap.J.

10. Y.N.Hayashida et all. 26th ICRC Salt Lake, USA, HE, (1998) ; astro-ph/9906056.

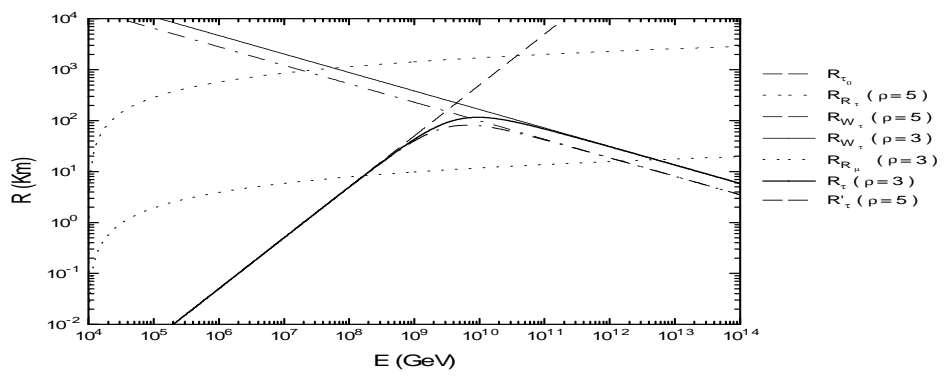

Fig. 1. The tau ranges as a function of the tau energy respectively for tau lifetime (dashed line) $R_{\tau_{o}}$, for tau radiation range $R_{R_{\tau}}$,(short dashed line above) and tau electroweak interaction range $R_{W_{\tau}}$, for two densities $\rho_{r}$ (long dashed lines, continuous) and their combined range $R_{\tau}$. Below the corresponding radiation range $R_{\mu}$ for muons (dotted line). 


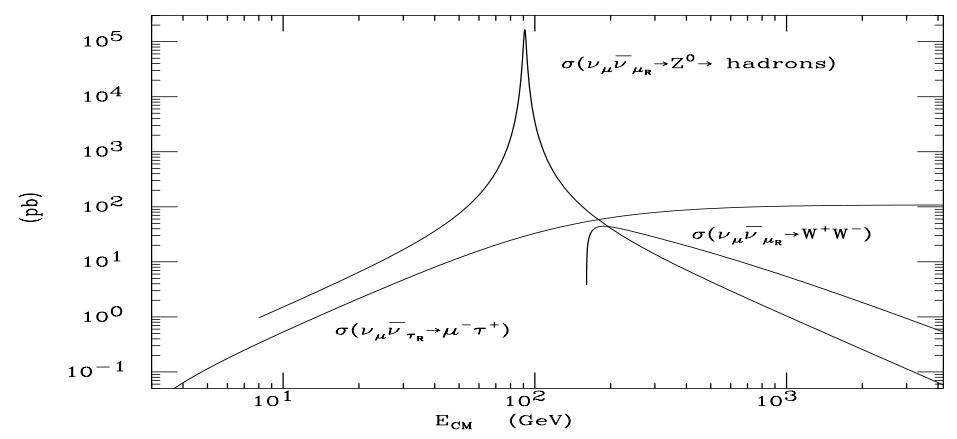

Fig. 2. The total cross sections for the UHE $\nu \bar{\nu}$ indicated processes as function of the center of mass energy (for a relic neutrino mass $m_{\nu}=10 \mathrm{eV}$ )(Fargion,Mele,Salis 1999)

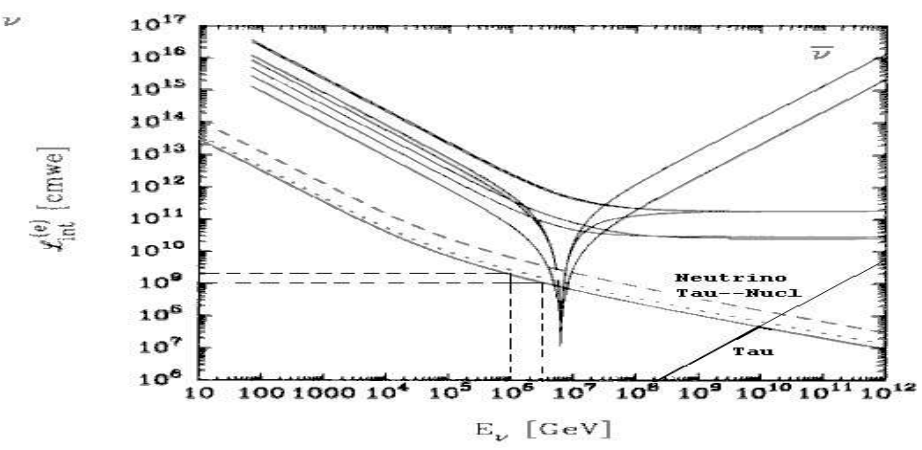

Fig. 3. The Gandhi et all (1998) UHE neutrino ranges as a function of UHE neutrino energy in Earth with overlapping $\bar{\nu}_{e} e, \nu_{\tau} N$ interactions; below the UHE $\tau$ range, as in Fig 1, at the same energies in matter (water).

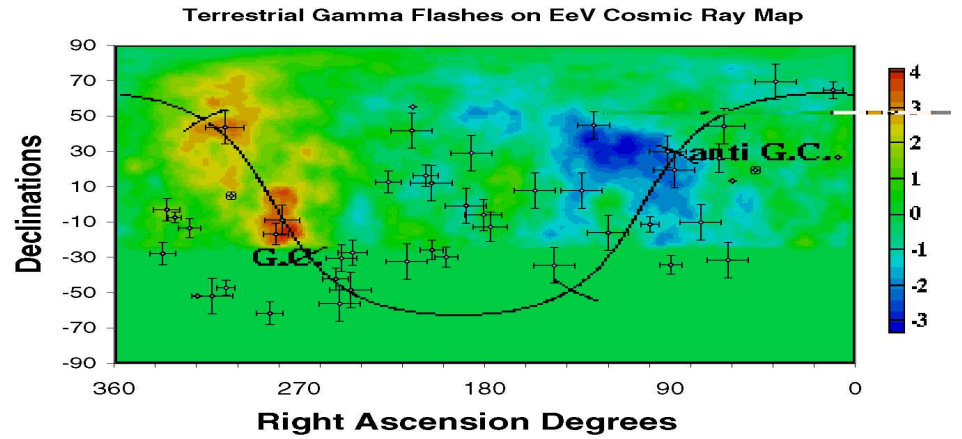

Fig. 4. Terrestrial Gamma Flash in celestial coordinate over UHECR diffused data by AGASA cosmic rays at EeV energies. 